\title{
Protectin conjugates in tissue regeneration 1 restores lipopolysaccharide-induced pulmonary endothelial glycocalyx loss via ALX/SIRT1/ NF-kappa B axis
}

Xin-Yang Wang ${ }^{\dagger}$, Xin-Yu Li ${ }^{\dagger}$, Cheng-Hua Wu, Yu Hao, Pan-Han Fu, Hong-Xia Mei, Fang Chen, Yu-Qiang Gong, Sheng-Wei Jin* and Hui Li*

\begin{abstract}
Background: Endothelial glycocalyx loss is integral to increased pulmonary vascular permeability in sepsis-related acute lung injury. Protectin conjugates in tissue regeneration 1 (PCTR1) is a novel macrophage-derived lipid mediator exhibiting potential anti-inflammatory and pro-resolving benefits.
\end{abstract}

Methods: PCTR1 was administrated intraperitoneally with $100 \mathrm{ng} / \mathrm{mouse}$ after lipopolysaccharide (LPS) challenged. Survival rate and lung function were used to evaluate the protective effects of PCTR1. Lung inflammation response was observed by morphology and inflammatory cytokines level. Endothelial glycocalyx and its related key enzymes were measured by immunofluorescence, ELISA, and Western blot. Afterward, related-pathways inhibitors were used to identify the mechanism of endothelial glycocalyx response to PCTR1 in mice and human umbilical vein endothelial cells (HUVECs) after LPS administration.

Results: In vivo, we show that PCTR1 protects mice against lipopolysaccharide (LPS)-induced sepsis, as shown by enhanced the survival and pulmonary function, decreased the inflammatory response in lungs and peripheral levels of inflammatory cytokines such as tumor necrosis factor-a, interleukin-6, and interleukin-1 $\beta$. Moreover, PCTR1 restored lung vascular glycocalyx and reduced serum heparin sulphate (HS), syndecan-1 (SDC-1), and hyaluronic acid (HA) levels. Furthermore, we found that PCTR1 downregulated heparanase (HPA) expression to inhibit glycocalyx degradation and upregulated exostosin-1 (EXT-1) protein expression to promote glycocalyx reconstitution. Besides, we observed that BAY1 1-7082 blocked glycocalyx loss induced by LPS in vivo and in vitro, and BOC-2 (ALX antagonist) or EX527 (SIRT1 inhibitor) abolished the restoration of HS in response to PCTR1.

Conclusion: PCTR1 protects endothelial glycocalyx via ALX receptor by regulating SIRT1/NF-KB pathway, suggesting PCTR1 may be a significant therapeutic target for sepsis-related acute lung injury.

Keywords: PCTR1, Glycocalyx, HPA, EXT-1, SIRT1

*Correspondence: jinshengwei69@163.com; judy198406@sina.com †Xin-Yang Wang and Xin-Yu Li contribute equally to this work Department of Anaesthesia and Critical Care, The Second Affiliated Hospital and Yuying Children's Hospital of Wenzhou Medical University, 109 Xueyuan Road, Wenzhou 325027, Zhejiang, People's Republic of China

\section{Background}

Sepsis is a common condition responsible for high morbidity and mortality worldwide [1]. Acute respiratory distress syndrome (ARDS), a severe sepsis complication, contributes to its high mortality [2]. Currently, there are no effective treatments or prevention available original author(s) and the source, provide a link to the Creative Commons licence, and indicate if changes were made. The images or other third party material in this article are included in the article's Creative Commons licence, unless indicated otherwise in a credit line to the material. If material is not included in the article's Creative Commons licence and your intended use is not permitted by statutory regulation or exceeds the permitted use, you will need to obtain permission directly from the copyright holder. To view a copy of this licence, visit http://creativecommons.org/licenses/by/4.0/. The Creative Commons Public Domain Dedication waiver (http://creativeco mmons.org/publicdomain/zero/1.0/) applies to the data made available in this article, unless otherwise stated in a credit line to the data. 
for sepsis-related lung injury [3-5]. The endothelium regulates the pathophysiology of lung injury [6]. The endothelial glycocalyx (EGL) primarily consists of glycosaminoglycans and proteoglycans, coating all healthy vascular endothelium [7]. Shedding of the endothelial glycocalyx is mainly driven by activation of heparan sulfate-specific heparanase, HPA [8]. Activated HPA promotes the degradation of the glycosaminoglycans and proteoglycans, and increases the level of glycocalyx components in the bloodstream, mainly including heparin sulphate (HS), hyaluronic acid (HA), and syndecan-1 (SDC-1) [8]. Exostosin-1 (EXT-1), a heparan sulfate elongation gene, is involved in glycocalyx synthesis and restitution, which is delayed in sepsis [9]. Damaged glycocalyx leads to an increase in permeability to proteins and fluids, causing interstitial leakage $[10,11]$. Therefore, it is critical to improve glycocalyx reconstitution for the treatment of sepsis.

Protectin conjugates in tissue regeneration 1 (PCTR1) is a newly discovered member of the specialized proresolving mediators (SPMs) from docosahexaenoic acid (DHA) belonging to the peptide-conjugated Protectins $[12,13]$. PCTR1 is identified and present in vivo before infection, and increases after LPS-induced inflammation, especially in inflammation resolution phrase [14]. Notably, PCTR1 not only enhances inflammation resolution by promoting macrophage efferocytosis and phagocytosis in infectious disease models but also improves tissue reparation $[15,16]$. However, the effect of PCTR1 on endothelial glycocalyx is still undefined.

Herein, the effects of PCTR1 on LPS-induced inflammation were investigated using a murine model for sepsis. Specifically, we examined its impact on survival rate, lung function, and inflammatory injury in the lungs. Next, we investigated its effect on lung vascular endothelial glycocalyx in mice. To obtain a more well-defined observation, LPS-induced injury in human umbilical vein endothelial cells (HUVECs) was performed to determine the direct effect of PCTR1 on endothelial glycocalyx. Finally, we determined the effect of the receptor, as well as pathways targeted by PCTR1 on LPS-induced glycocalyx loss to gain insights into the underlying mechanisms.

\section{Methods}

\section{Animal preparation}

Specific pathogen-free (SPF) adult male C57BL/6 mice (6-8 wk) were bought from SLAC Laboratory Animal CO. (Shanghai, China). Before the experiments, the mice were kept in standard cages in a house controlled to a $12 \mathrm{~h} \mathrm{light/dark} \mathrm{cycle} \mathrm{with} \mathrm{temperature} \mathrm{maintained}$ at $22-24{ }^{\circ} \mathrm{C}$, humidity at $50-60 \%$ and SPF environment in Wenzhou Medical University. The mice were allowed free access to water and food. All animal procedures conformed to the Guide for the Care and Use of Laboratory Animals. This was approved by the Animal Studies Ethics Committee of Wenzhou Medical University.

\section{Animal experimental groups}

Mice were intraperitoneally injected with LPS (15 mg/ kg, Escherichia coli, serotype 055: B5; Sigma, Saint Louis, MO, USA) for survival experiments and LPS (10 mg/kg) for other experiments. The effect of PCTR1 (Cayman Chemical, Ann Arbor, MI, USA) on LPS-induced sepsis model was determined by establishing four experimental groups: control group, LPS group, LPS + PCTR1 group (LPS and PCTR1 were given simultaneously), PCTR1 group. For the PCTR1 and the control groups, the mice were intraperitoneally injected with PCTR1 $100 \mathrm{ng} /$ mouse or an equal saline volume. Next, to determine the mechanism of PCTR1, four groups were established: control group, LPS group, LPS + PCTR1 group, LPS + PCTR1 + BOC-2 group, LPS + PCTR1 + EX527 group, and LPS + BAY11-7082 group. Mice were administered with BOC-2 $(600 \mathrm{ng} / \mathrm{kg}$, Biomol-Enzo Life Sciences, Farmingdale, NY), EX527 (10 mg/kg, MEC, Shanghai, China), and BAY11-7082 (20 mg/kg, Selleck, Houston, TX, USA) intraperitoneally following LPS administration. The mice were anesthetized with $1 \%$ pentobarbital and sacrificed $6 \mathrm{~h}$ later. We obtained blood samples via the ophthalmic artery from mice that survived in each group, and obtained lung specimens.

\section{Cell culture and experimental groups}

Human umbilical vein endothelial cells (HUVECs) were purchased from SGST (China). The cells were suspended in a DMEM medium containing 10\% FBS and cultured in $25 \mathrm{~cm}^{2}$ flasks placed in an incubator controlled at 5\% $\mathrm{CO}_{2}$ at $37{ }^{\circ} \mathrm{C}$. In all experiments, HUVECs were added to the wells of six-well plates at equal concentrations and were further sub-divided into six groups: control group, LPS group, LPS + PCTR1 group (LPS and PCTR1 were given simultaneously), LPS + PCTR1 + DMSO group (DMSO was used as the solvent for EX527), LPS + PCTR1 + EX527 group and LPS + BAY11-7082 group. DMSO, EX527 $(1 \mu \mathrm{M})$, and BAY11-7082 $(1 \mu \mathrm{M})$ were added to cells for $24 \mathrm{~h}$ followed by LPS $(1 \mu \mathrm{g} / \mathrm{ml})$ and/or PCTR1 (100 nM) stimulation. The LPS or PCTR1 we used in vitro are the same one with we used in vivo.

\section{Invasive evaluation of respiratory mechanics}

The lung function assay was conducted using a flexiVent system (Scireq, Montreal, QC, Canada), as described previously [17]. In brief, mice were anesthetized with $90 \mathrm{mg} /$ $\mathrm{kg}$ pentobarbital sodium injected intraperitoneally and then tracheotomized. Vecuronium bromide was administered into each mice via intravenous injection, followed 
by mechanical ventilation using a computer-moderated small-animal ventilator. A model of deep inflation was used to record the inspiratory capacity (IC). Cst (quasistatic compliance) was assessed based on the PV curves.

\section{Lung morphology}

Left lungs were excised after anesthetization, and fixed overnight with $4 \%$ paraformaldehyde at room temperature. This was followed by staining of 5- $\mu \mathrm{m}$ sections using hematoxylin and eosin (HE) and microscopic examination. The lung injury score was determined based on the level of infiltration of inflammatory cells, hyperemia, and the thickness of the alveolar wall.

\section{Lung vascular permeability assay}

The evans blue dye (EBD) extravasation was utilized to assess pulmonary vascular permeability. Five and a half hours following LPS administration, EBD $(20 \mathrm{mg} / \mathrm{kg})$ was injected through the caudal vein. After the circulation of the dye for $30 \mathrm{~min}$, perfusion of lungs was performed with saline $(25 \mathrm{ml})$ under anaesthesia. Subsequently, the lungs were excised, blotted dry, weighed, and homogenized in formamide. After overnight extraction, the tissue fluid was centrifugated for $10 \mathrm{~min}$ at $12,000 \times \mathrm{g}$. A microplate reader was used to read the EBD concentration of the resultant supernatant at $620 \mathrm{~nm}$ absorbance.

\section{Wet-to-dry lung weight ratio}

The index of pulmonary edema was determined from the wet-to-dry (W/D) lung weight ratio. Portions of the harvested wet left lungs were weighed and heated in an oven at $60{ }^{\circ} \mathrm{C}$ for $48 \mathrm{~h}$. The W/D ratio was then determined after re-weighing the portions as dry weight.

\section{ELISA}

Blood samples were obtained to determine level serum inflammatory cytokines, as well as the degradation products of glycocalyx in circulation. The blood was collected using the orbital sinus extraction method under anaesthesia. The serum was isolated from the blood samples for ELISA tests. Serum levels of cytokines including TNF- $\alpha$, IL-1 $\beta$, and IL- 6 and glycocalyx-related proteins including HS, SDC-1, and HA were quantified using R\&D systems and Boyun biotech ELISA kits following protocols provided by the manufacturer.

\section{Western blot}

Lung tissues were homogenized with lysis buffer (RIPA: $\mathrm{PMSF}=1: 1)$ to obtain protein samples. The tissue homogenates were ultrasonicated and then centrifuged for $30 \mathrm{~min}$ at $12,000 \times \mathrm{g}$. The concentration of proteins in the supernatants was measured using the BCA kit. An equal amount of protein from each group was loaded onto 10\% SDS-PAGE gels and resolved. The proteins were electro-transferred onto PVDF membranes. After being blocked with $10 \%$ milk for $2 \mathrm{~h}$, membranes were incubated with the primary antibodies: EXT-1 (1:2000, GeneTex, Irvine, CA, USA), HPA (1:1000, Abcam, Cambridge, MA, USA), p65 (1:1000, Abcam, Cambridge, MA, USA), p-p65 (1:1000, Abcam, Cambridge, MA, US), SIRT1 (1:1000, Abcam, Cambridge, MA, USA), and $\beta$-actin (1:1000, BOYUN, Shanghai, China) overnight at $4{ }^{\circ} \mathrm{C}$. Next, the membranes were washed thrice and incubated with secondary antibody (1:3000, BOYUN, Shanghai, China) at room temperature for $1 \mathrm{~h}$ followed by another three-times wash. The protein bands were analyzed by Image Quant LAS 4000 mini (GE Healthcare Bio-Sciences AB, Uppsala, Sweden). The band intensity was analyzed with ImageJ.

\section{Immunofluorescence}

Immunofluorescence was carried out using lung tissues and HUVECs. Lung sections were prepared for immunofluorescence after deparaffinized, dehydrated and antigen retrieval. This was followed by fixation of the HUVECs in $4 \%$ paraformaldehyde. Subsequently, the fixed cells and tissue samples were mounted on the cover glass, blocked with donkey serum (Solarbio, Beijing, China) and probed with HSPG2-antibody (1:200). After rinsing thrice with PBS, the fixed cells and tissue sections were incubated with the secondary antibody $(1: 200)$ at $37{ }^{\circ} \mathrm{C}$ for $1 \mathrm{~h}$ and further incubated with DAPI (Abcam) for $5 \mathrm{~min}$. Eventually, both fixed cells and sectioned tissues were covered with an antifade mounting medium (Solarbio, Beijing, China) for fluorescence microscopy (Leica).

\section{Data analysis}

The mean values are presented as the mean $\pm \mathrm{SD}$ and were analyzed with GraphPad Prism 7.0 software. Mean values of groups were compared one-way ANOVA followed by Tukey's post hoc test for multiple comparisons. Kaplan-Meier analysis was conducted to evaluate survival and a log-rank (Mantel-Cox) test was employed to determine statistical significance. $P<0.05$ was considered statistically significant.

\section{Results}

PCTR1 improves survival and lung function in mice after LPS administration

We first determine the effect of PCTR1 on septic mice by survival curve analysis. PCTR1 significantly promoted survival in mice subjected to LPS $(15 \mathrm{mg} / \mathrm{kg})$ injected (Fig. 1a). Next, we performed a lung function test on LPS (10 mg/kg)-induced endotoxemia mice. Compared with the saline group, mice with LPS challenge showed a 

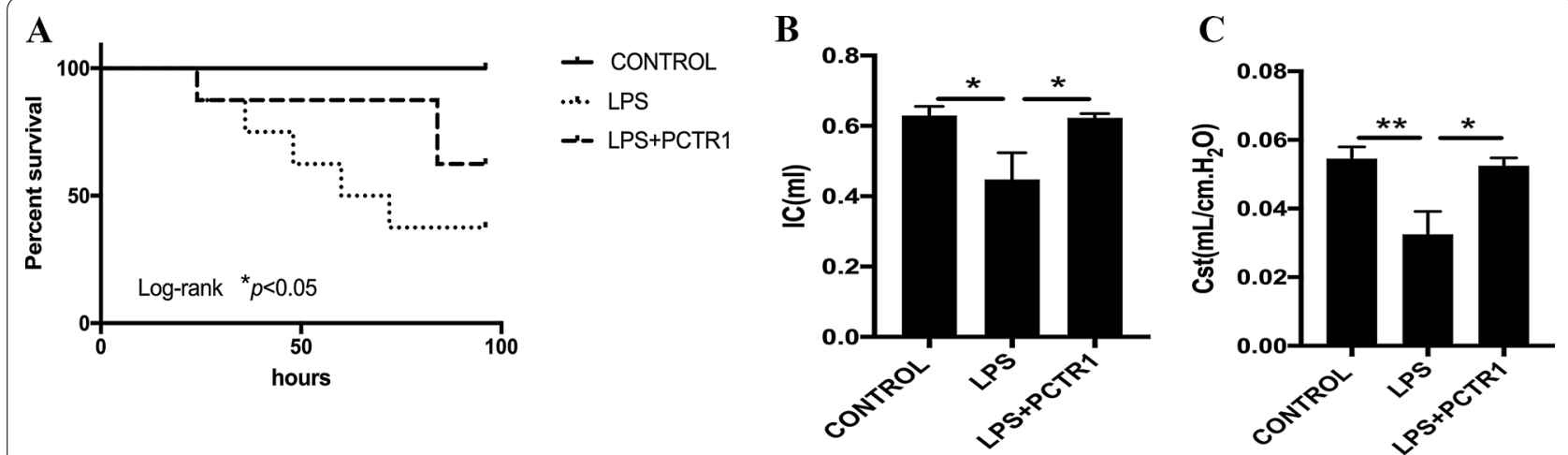

Fig. 1 PCTR1 improves survival and lung function in mice after LPS administration. a Kaplan-Meier survival curves for the LPS (15 mg/kg) with or without PCTR1 (100 ng/mouse) treated mice $(n=8)$. Lung function of each group mice was measured by the flexiVent apparatus at $6 \mathrm{~h}$ after LPS $\left(10 \mathrm{mg} / \mathrm{kg}\right.$ ) with or without PCTR1 (100 ng/mouse) challenge. b, c IC (Inspiratory Capacity) and Cst (Static Compliance) are shown $(n=5) .{ }^{*} P<.05$, ${ }^{* *} P<.01$

statistically significant reduction in IC and Cst, whereas PCTR1 enhanced IC and Cst significantly (Fig. 1b, c).

\section{PCTR1 alleviates LPS-stimulated lung inflammatory response in mice}

The histology in the control group showed normal lung conditions in mice. Notably, lung tissues in the LPS group were remarkably damaged, involving alveolar disarray, as well as infiltration level of inflammatory cells as indicated by increases in lung injury scores unlike mice in the control group. There was no marked change in morphologic features in the LPS + PCTR1 group. There was no remarkable difference between the control and the PCTR1 group (Fig. 2a, b). Accordingly, the peripheral levels of inflammatory cytokines, e.g., TNF- $\alpha$, IL-1 $\beta$, and IL- 6 were remarkably upregulated in the LPS group relative to the control group and were lower in the PCTR1 treatment group compared with the LPS group (Fig. 2ce). Furthermore, to determine pulmonary vascular permeability in mice, the EBD test and W/D ratio of lung tissues were assessed. As expected, PCTR1 significantly inhibited LPS induced EBD and W/D increase of lung tissues (Fig. 2f, g).

\section{PCTR1 restores LPS-induced endothelial glycocalyx damage in mice}

On account of the importance of endothelial glycocalyx in maintaining vascular permeability, we tested the effect of PCTR1 on LPS-induced endothelial glycocalyx damage. Our results demonstrated that PCTR1increased the expression of HS and SDC-1 in lungs after LPS challenge (Fig. 3a-d). Accordingly, PCTR1 reduces the peripheral concentration of $\mathrm{HS}, \mathrm{SDC}-1$ and $\mathrm{HA}$ in septic mice (Fig. 3e-g).

\section{PCTR1 regulates HPA and EXT-1 expression} in LPS-triggered sepsis in mice

The loss of endothelial glycocalyx induced pulmonary vascular hyperpermeability. HPA and EXT-1 are key enzymes involving in maintaining endothelial glycocalyx integrity via regulating degradation and reconstitution, respectively. PCTR1 decreased HPA expression and increased EXT-1 expression in LPS-induced septic mice. Meanwhile, the lower SIRT1 expression and its downstream, the higher NF-kB p65 phosphorylation (p-p65) expression were found in LPS group mice; by contrast, PCTR1 inhibited those changes in mice after LPS challenge (Fig. 4a, b). Together with the finding that, we postulate that PCTR1 might restore both HPA- and EXT-1-mediated endothelial glycocalyx dynamic balance through enhancing SIRT1 expression. As shown in our results, the inhibition of NF-kB blocked the effect of LPS on HPA and EXT-1, whilst the inhibition of SIRT1 blocked the effect of PCTR1 on HPA and EXT-1. Furthermore, we found that the effect of PCTR1 was abolished in the presence of BOC-2, the GFPR inhibitor (Fig. 4c, d).

\section{PCTR1 protects the endothelial glycocalyx by modulating SIRT1/NF-KB pathways via ALX receptor}

To determine whether PCTR1 restores endothelial glycocalyx via ALX/SIRT1/NF-kB, we assessed the HS expression in lung sections of each group by immunofluorescence. As expected, the effect of LPS on HS was inhibited in the presence of BAY11-7082, and the effect of PCTR1 on HS was abolished in the presence of BOC- 2 or EX527 (Fig. 5a, b). HUVEC endothelial cell was used for evaluating the effect of PCTR1 on endothelial glycocalyx in vitro. Consistent with the experiment results in vivo, we found that BAY11-7082 inhibited LPS-induced HS 


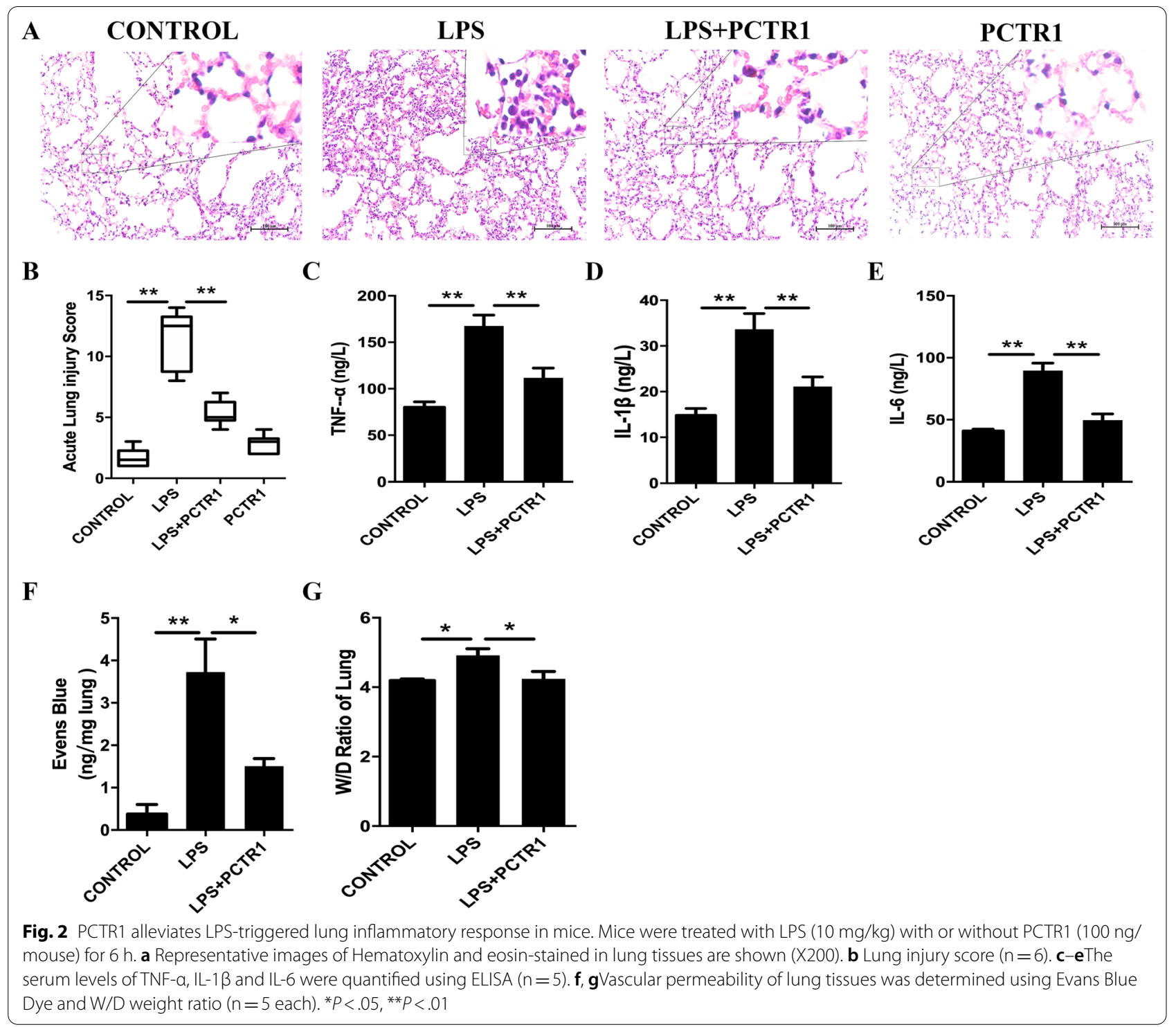

loss, and EX527 inhibited PCTR1-induced HS restoration in HUVECs after LPS stimulation (Fig. 5c, d).

\section{Discussion}

This study results provided research evidence of the protective effects of PCTR1 in a murine model of LPS injection. We first observed that PCTR1inhibited the infiltration of neutrophils, the generation of TNF- $\alpha$, IL- $1 \beta$, as well as IL- 6 and LPS-provoked hyperpermeability in the lungs, with the outcome of enhanced survival and lung function. Next, we demonstrated that PCTR1 reduced lung endothelial glycocalyx degradation and improved glycocalyx reconstitution after LPS administration. Furthermore, we found PCTR1 decreased glycocalyx degradation factor HPA and increased glycocalyx key synthetase EXT-1 via ALX/SIRT1/NF-kB, restoring endothelial glycocalyx consequently (Fig. 6).

PCTR1 is a new endogenous mediator with the potential to promote inflammation resolution via $G$ protein-coupled receptors(ALX receptor) [18]. It was demonstrated that PCTR1 is increased to enhance macrophage recruitment and phagocytosis during the inflammation resolution phase [14]. Moreover, PCTR1 generation in peritoneal lavage is decreased in vagotomized mice, suggesting that PCTR1 is controlled by local vagal to improve inflammation resolution [15]. Our previous studies demonstrated that PCTR1 reduced LPS-induced inflammation in mice via regulating linoleic acid metabolism, and improved pulmonary edema fluid clearance by activating the sodium channel 

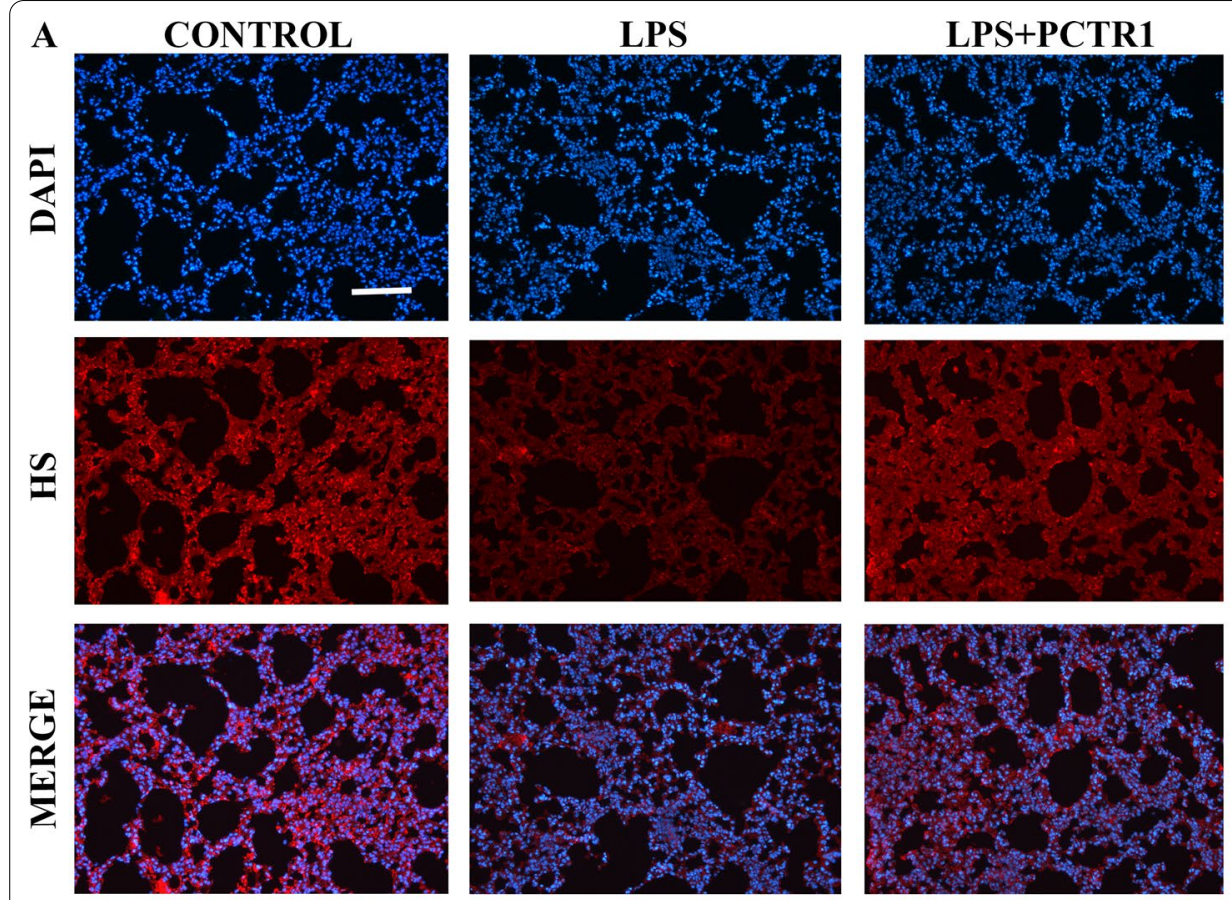

D
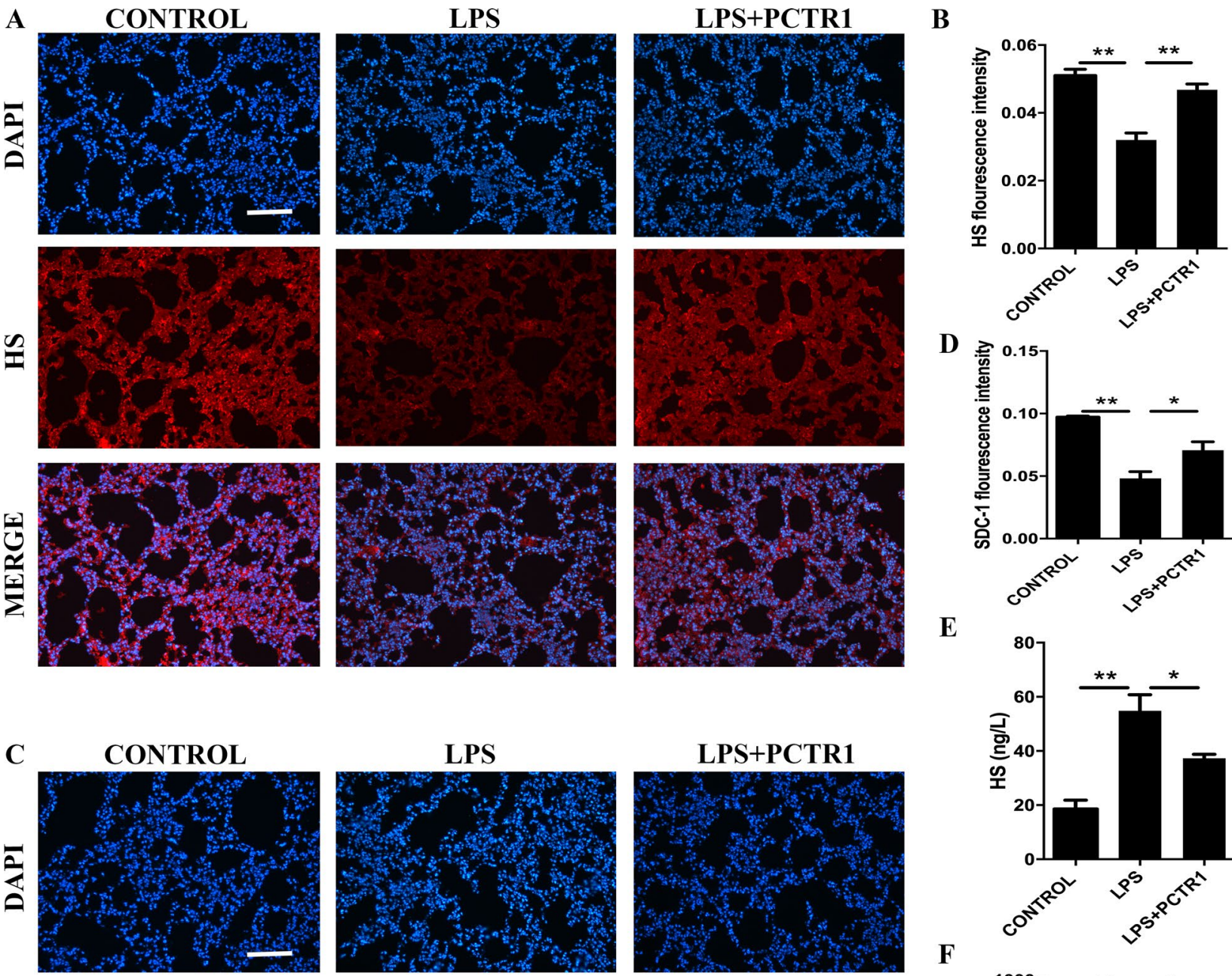

$\mathbf{E}$
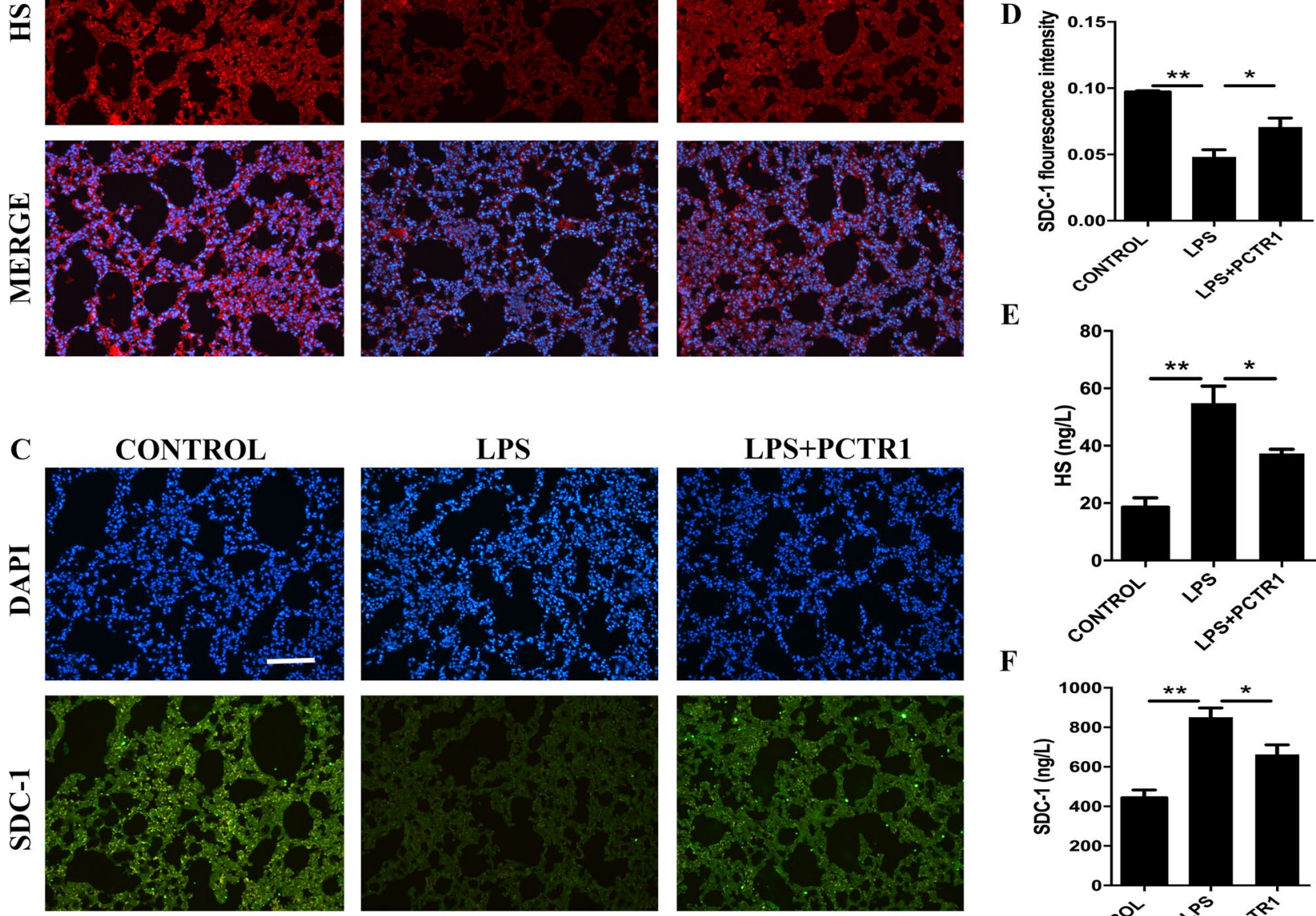

F
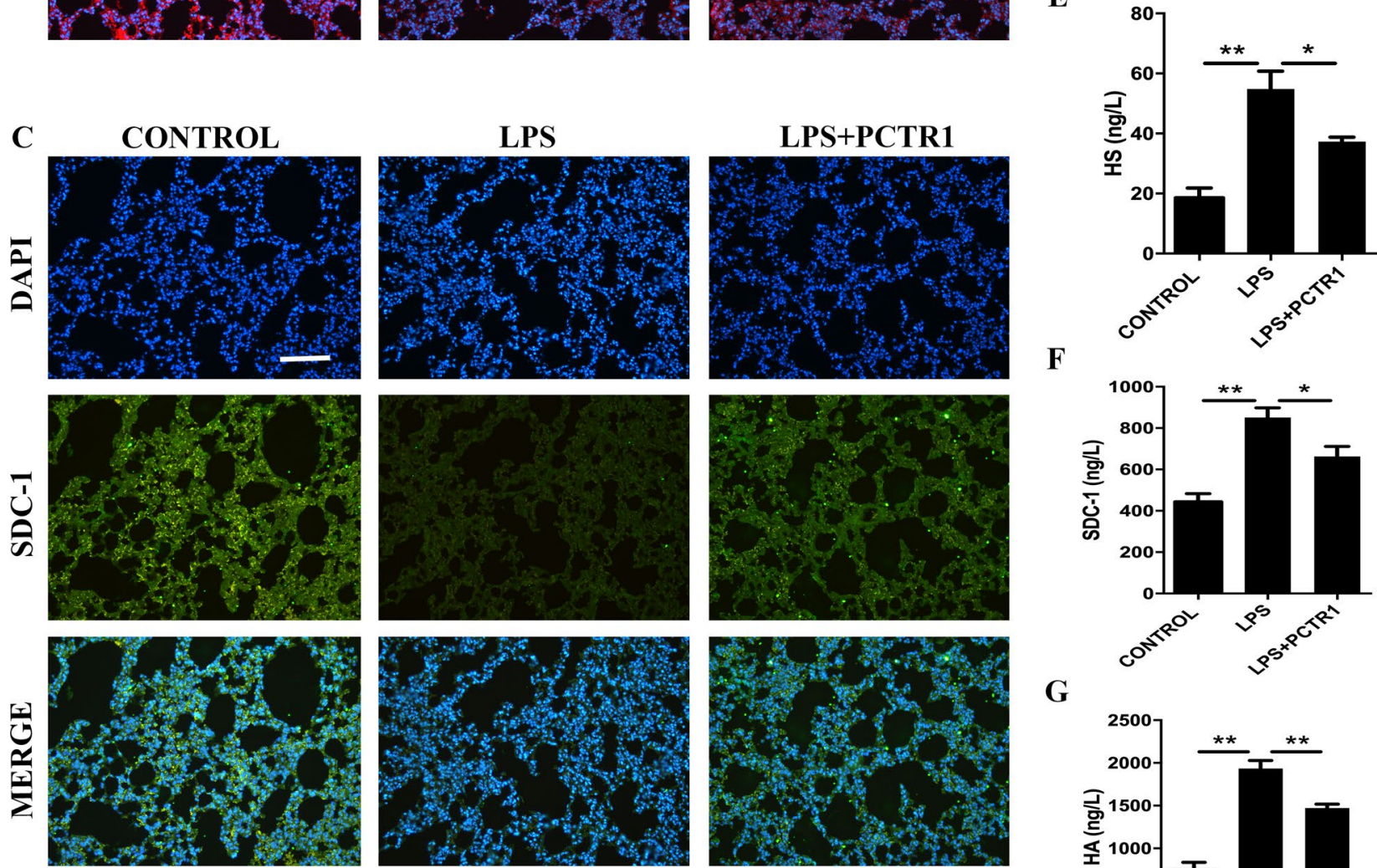

G

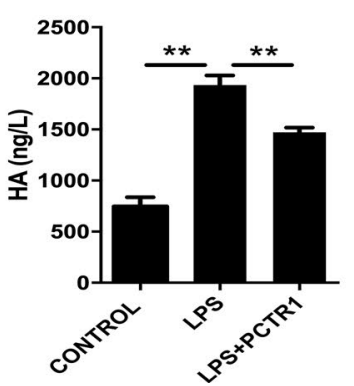

Fig. 3 PCTR1 restores LPS-triggered endothelial glycocalyx damage in mice. LPS (10 mg/kg) with or without PCTR1 (100 ng/mouse) was injected to mice for $6 \mathrm{~h}$. Representative image and densitometry of immunofluorescence of HS $(\mathbf{a}, \mathbf{b})$ and SDC-1 $(\mathbf{c}, \mathbf{d})$ in the lungs. Scar bar: $50 \mu \mathrm{m}$. $\mathbf{e}-\mathbf{g}$ After collecting blood from the eyeballs, the serum levels of HS, SDC-1 and HA were quantified by ELISA. ${ }^{*} P<.05,{ }^{*} P<.01, \mathrm{n}=5$ 


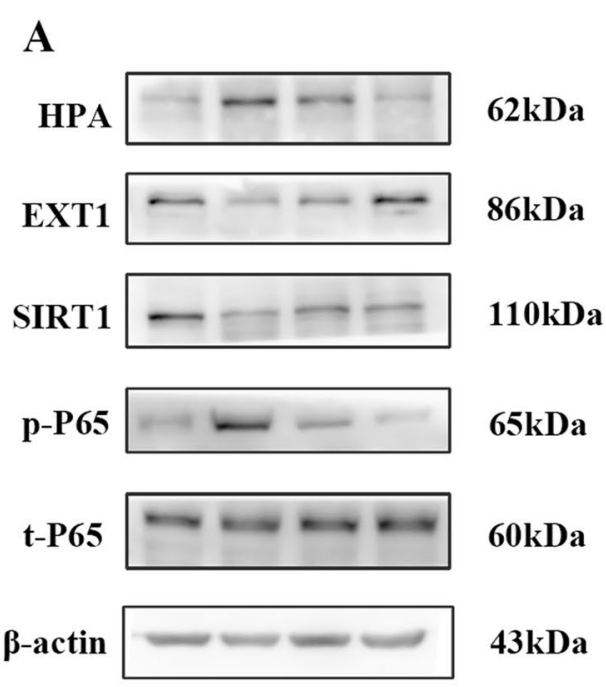

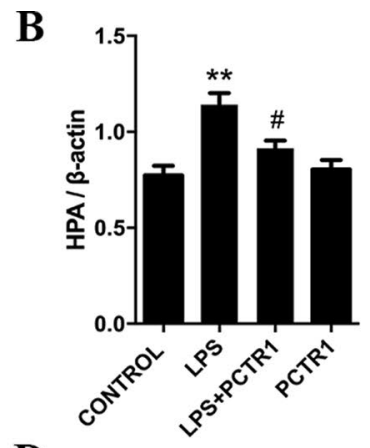

D

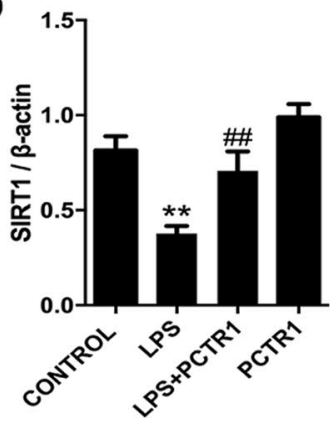

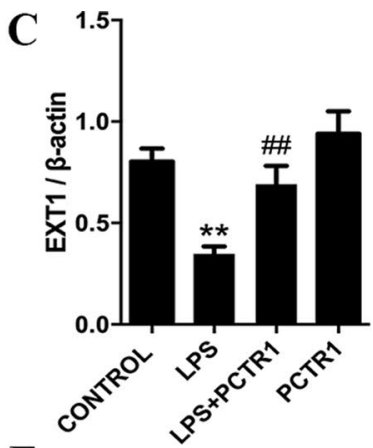

$\mathbf{E}$

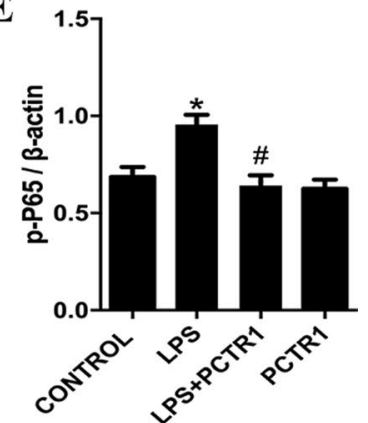

F

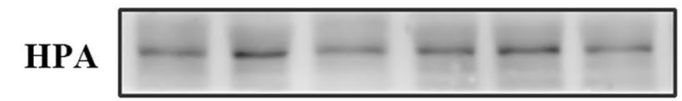

$62 \mathrm{kDa}$

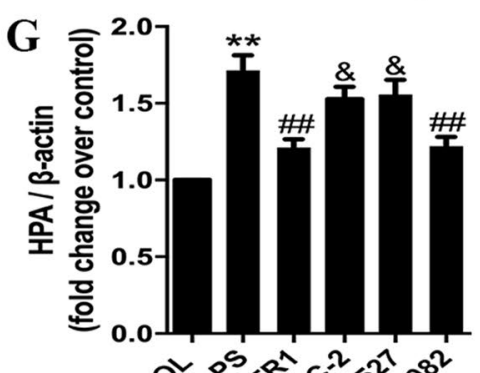

\section{EXT1}

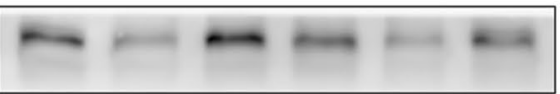

$\beta$-actin

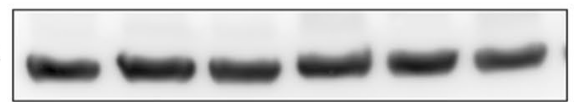

LPS -++++

PCTR1 - -+++

BOC-2 - $-\quad+-$

EX527 - $-\quad-\quad+\quad+$
86kDa

$43 \mathrm{kDa}$

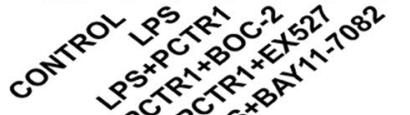

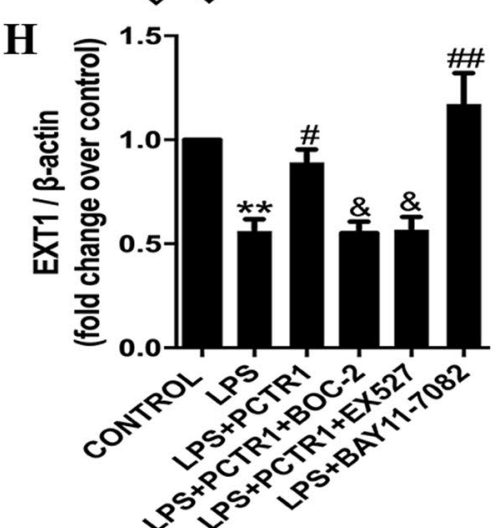

Fig. 4 PCTR1 regulates HPA and EXT-1 expression in LPS- triggered sepsis in mice. a-e Mice were treated with LPS (10 mg/kg) with or without PCTR1 (100 ng/mouse) for $6 \mathrm{~h}$. The levels of HPA, EXT-1, SIRT1 and p65 in the lung tissue were quantified by western blot. f-h BOC-2 (ALX receptor inhibitor, $600 \mathrm{ng} / \mathrm{kg}$ ) or EX527 (SIRT1 inhibitor, $10 \mathrm{mg} / \mathrm{kg}$ ) or BAY11-7082 (NF-KB inhibitor, $20 \mathrm{mg} / \mathrm{kg}$ ) was injected $1 \mathrm{~h}$ followed by LPS (10 mg/ $\mathrm{kg}$ ) with or without PCTR1 (100 ng/mouse). The protein levels of HPA and EXT-1 were measured by western blot. ${ }^{*} P<.05$, ${ }^{* *} P<.01$ relative to the CONTROL group, ${ }^{\#} P<.05,{ }^{\#} P<.01$ relative to the LPS group, ${ }^{\&} P<.05$, relative to the LPS $+P C T R 1$ group. $n=6$ 

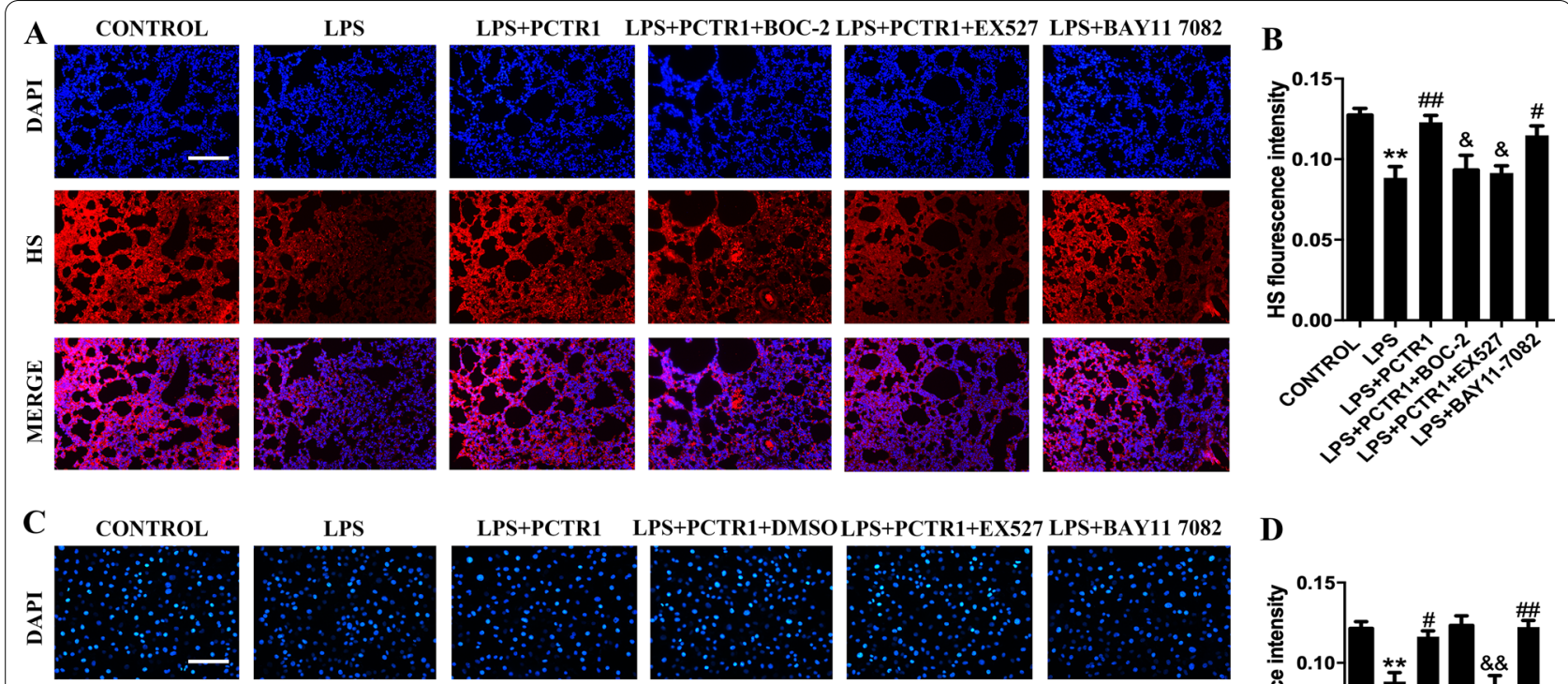

LPS
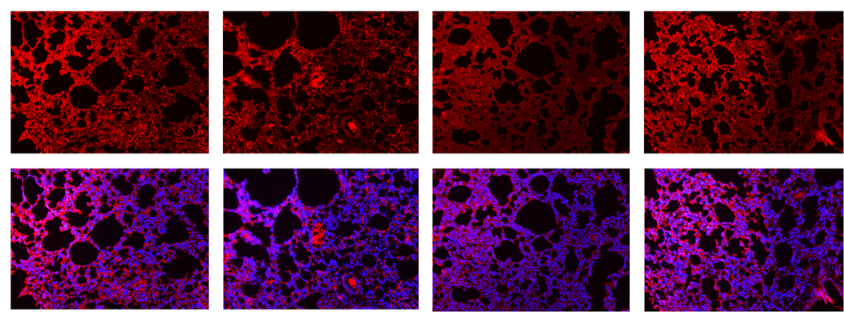

LPS+PCTR1 LPS+PCTR1+DMSOLPS+PCTR1+EX527 LPS+BAY11 7082
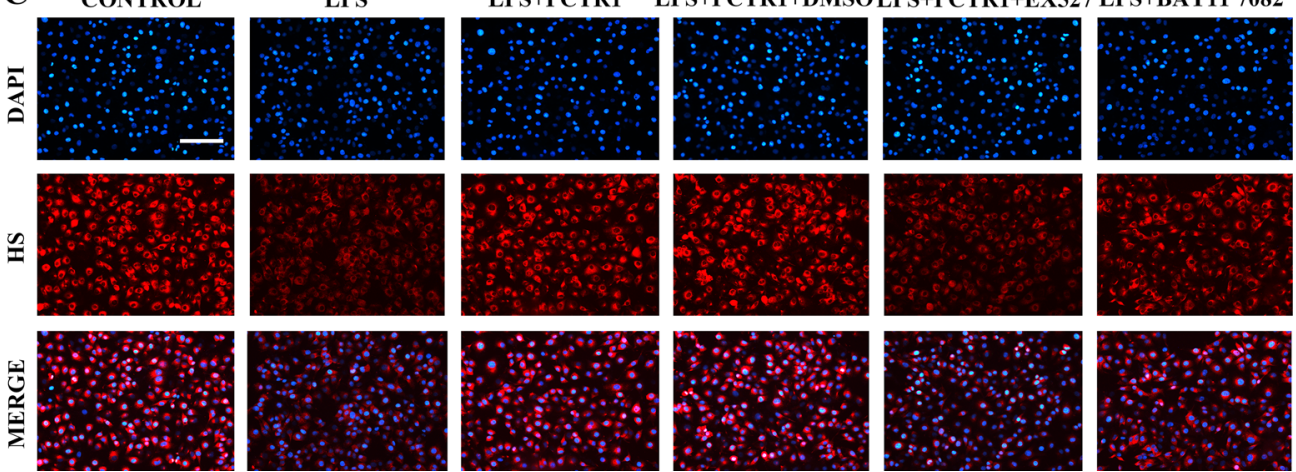

D

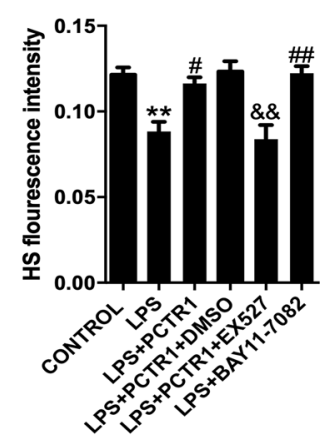

Fig. 5 PCTR1 protects the endothelial glycocalyx by modulating SIRT1/NF-KB pathways via ALX receptor. The level of HPA, EXT-1, SIRT1 and p65 in lung tissues was quantified using western blotting assay. BAY11-7082 (NF-KB inhibitor, $20 \mathrm{mg} / \mathrm{kg}$ ) or EX527 (SIRT1 inhibitor, $10 \mathrm{mg} / \mathrm{kg}$ ) or BOC-2(ALX receptor inhibitor, $600 \mathrm{ng} / \mathrm{kg}$ ) was injected $1 \mathrm{~h}$ followed by LPS (10 mg/kg) with or without PCTR1 (100 ng/mouse) for 6 h. (a, b) The expression of HS in tissue was examined by immunofluorescence. HUVECs were administered with $1 \mu \mathrm{g} / \mathrm{ml}$ LPS and $100 \mathrm{nM}$ PCTR1 for $6 \mathrm{~h}$. After treatment with EX527 or DMSO, HS in HUVECS was determined by immunofluorescence $(\mathbf{c}, \mathbf{d})$. Scar bar $=50 \mu \mathrm{m}$. ${ }^{* *} P<.01$ in comparison to the CONTROL group, ${ }^{\#}$ $P<.05,{ }^{\# \#} P<.01$ in comparison to the LPS group, ${ }^{\&} P<.05,{ }^{\&} \&<.01$, in comparison to the LPS + PCTR1 group. $\mathrm{n}=6$

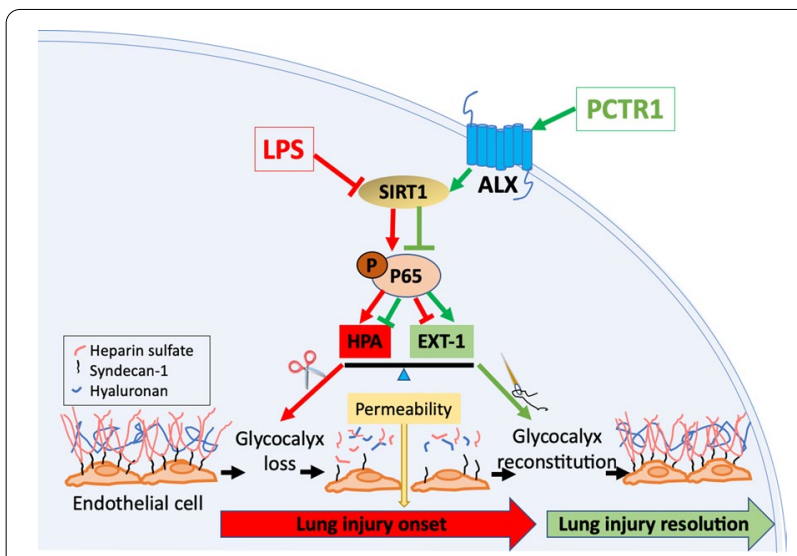

Fig. 6 PCTR1 restores Lipopolysaccharide-induced pulmonary endothelial glycocalyx via ALX/SIRT1/NF-kappa B axis in mice. The effect of LPS was shown in red, whilst the effect of PCTR1 was shown in green. ALX: a G protein-coupled receptor; LPS: Lipopolysaccharides; PCTR1: Protectin conjugates in tissue regeneration 1; SIRT1: sirtuin 1; HPA: heparanase; EXT-1: exostosin-1 and lymphatic drainage in LPS-triggered rat model [19, 20]. Similarly, we also observed that PCTR1 improved lung function and lung vascular leakage after LPS challenge in mice.

Increasing evidence demonstrates that the intact glycocalyx contributes to the maintenance of vascular integrity and leukocyte trafficking [21]. Whilst, after an acute injury, glycocalyx is degraded by HPA, and the level of glycocalyx components such as HS, SDC-1 and HA is elevated in the bloodstream, which could predict outcomes of patients with sepsis-induced organ dysfunction, including ARDS [22, 23]. In sepsis, EXT-1 expression is decreased, and glycocalyx reconstitution is delayed, which increases sepsis severity [24]. Previous studies has reported that enhancing endothelial fibroblast growth factor receptor 1 signaling improves EXT-1-mediated pulmonary glycocalyx reconstitution in sepsis $[10,25]$. Thus, endothelial glycocalyx protection has been considered as a therapeutic strategy to treat sepsis. Herein, we found that PCTR1 unregulated EXT-1 expression and downregulated HPA expression, 
promoting glycocalyx reconstitution in sepsis model via ALX receptor consequently.

It has been demonstrated that SIRT1 is a negative regulator of inflammation [26]. Previous studies reported that endothelial glycocalyx decreased in vivo and in vitro with a defect in SIRT1 deacetylase activity [27, 28]. Consistently, we found that the protective effect of PCTR1 on glycocalyx was abolished in the presence of the inhibitor of SIRT1. SIRT1 has been reported to suppress inflammatory responses by inhibiting NF- $\mathrm{kB}$ signaling [29]. SIRT1 not only stimulates oxidative energy production but also inhibits transcription by deacetylating p 65 and suppresses inflammation [30,31]. On the other hand, the increases of HPA expression in various inflammation and cancer models with the increase of NF- $\mathrm{kB}$ P65 phosphorylation [32]. It is reported that NF- $\mathrm{kB}$ inhibitor attenuated HPA expression [32, 33]. In our study, we found that LPS increased HPA expression and decreased EXT-1 expression via activating NF- $\mathrm{KB}$, degrading glycocalyx consequently in vivo. HUVEC was considered as a common choice for the endothelial experiment in vitro [34]. Consisitent results were also obtained from the experiment in HUVEC. Whilst, PCTR1 induced SIRT1 expression and reduced NF- $\mathrm{KB}$ phosphorylation after LPS administration and reversed the effect of LPS on glycocalyx in vivo and in vitro.

\section{Conclusions}

This study indicates that PCTR1 reduces endothelial glycocalyx loss and improves glycocalyx reconstitution by modulating glycocalyx key enzymes including HPA and EXT-1 through ALX/SIRT1/NF- $k B$ cascade after LPS treatment. The preservation of the glycocalyx attenuates lung inflammation response and vascular endothelial hyperpermeability, and further restores lung function and survival rate after LPS administration in mice. This study not only identifies a protective action of PCTR1 in LPS-induced sepsis mice, but the results may also open a new therapeutic strategy with PCTR1 for other vascular-related diseases.

\section{Abbreviations \\ PCTR1: Protectin conjugates in tissue regeneration 1; LPS: Lipopolysac- charide; HS: Heparin sulphate; SDC-1: Syndecan-1; HA: Hyaluronic acid; HPA: Heparanase; EXT-1: Exostosin-1; ARDS: Acute respiratory distress syndrome; EGL: Endothelial glycocalyx; SPMs: Specialized pro-resolving mediators; DHA: Docosahexaenoic acid; SPF: Specific pathogen-free; HUVECs: Human umbilical vein endothelial cells; IC: Inspiratory capacity; Cst: Quasistatic compliance; HE: Hematoxylin and eosin; EBD: Evans blue dye; W/D: Wet-to-dry; GPCR: G protein-coupled receptors; SIRT1: Sirtuin 1.}

\section{Acknowledgements}

We thank Xiao-Ying Huang for lung function test. We thank all mice in our experiment for helping us to identify the hypothesis.

\section{Authors' contributions}

X-YW: Investigation. X-YL: Investigation. C-HW: Investigation. YH: Methodology, Software. P-HF: Methodology, Software. H-XM: Investigation. FC: Investigation. Y-QG: Conceptualization. S-WJ: Conceptualization, Funding acquisition, Validation. HL: Conceptualization, Writing, Validation, Funding acquisition. All authors read and approved the final manuscript.

\section{Funding}

The study was supported by funds from the Zhejiang Health Innovation Talent Project (Nos.2021RC094), Natural Science Foundation of Zhejiang Province (Nos. LQ20H150003, LQ19H150001, LY19H150002), Zhejiang Science and Technology department key research and development program (No. 2019C03011), Wenzhou Science and Technology Bureau Project (Nos. Y20190087, Y20190118).

\section{Availability of data and materials}

All supporting data are available from the corresponding author upon reasonable request.

\section{Declarations}

\section{Ethics approval and consent to participate}

All animal experiments in this study were performed in accordance with the Guide for the Care and Use of Laboratory Animals of Wenzhou Medical University.

\section{Consent for publication}

Not applicable.

\section{Competing interests}

There are no conflict of interest to declare.

Received: 30 October 2020 Accepted: 30 June 2021

Published online: 03 July 2021

References

1. Cecconi M, Evans L, Levy M, Rhodes A. Sepsis and septic shock. Lancet. 2018:392:75-87.

2. Englert JA, Bobba C, Baron RM. Integrating molecular pathogenesis and clinical translation in sepsis-induced acute respiratory distress syndrome. JCl Insight. 2019;4:1

3. Patel VJ, Biswas Roy S, Mehta HJ, Joo M, Sadikot RT. Alternative and natural therapies for acute lung injury and acute respiratory distress syndrome. Biomed Res Int. 2018;2018:2476824.

4. Griet M, Zelaya H, Mateos MV, Salva S, Juarez GE, de Valdez GF, Villena J, Salvador GA, Rodriguez AV. Soluble factors from Lactobacillus reuteri CRL1098 have anti-inflammatory effects in acute lung injury induced by lipopolysaccharide in mice. PLOS ONE. 2014;9:e110027.

5. Leaf DE, Waikar SS. Rosuvastatin for sepsis-associated ARDS. N Engl J Med. 2014;371:968.

6. Huber-Lang M, Lambris JD, Ward PA. Innate immune responses to trauma. Nat Immunol. 2018;19:327-41.

7. Yang Y, Schmidt EP. The endothelial glycocalyx: an important regulator of the pulmonary vascular barrier. Tissue Barriers. 2013;1:1.

8. Chappell D, Jacob M, Rehm M, Stoeckelhuber M, Welsch U, Conzen P, Becker BF. Heparinase selectively sheds heparan sulphate from the endothelial glycocalyx. Biol Chem. 2008;389:79-82.

9. Mooij HL, Cabrales P, Bernelot Moens SJ, Xu D, Udayappan SD, Tsai AG, van der Sande MA, de Groot E, Intaglietta M, Kastelein JJ, et al. Loss of function in heparan sulfate elongation genes EXT1 and EXT 2 results in improved nitric oxide bioavailability and endothelial function. J Am Heart Assoc. 2014;3:e001274.

10. LaRiviere WB, Schmidt EP. The pulmonary endothelial glycocalyx in ARDS: a critical role for heparan sulfate. Curr Top Membr. 2018;82:33-52.

11. Schmidt EP, Yang Y, Janssen WJ, Gandjeva A, Perez MJ, Barthel L, Zemans RL, Bowman JC, Koyanagi DE, Yunt ZX, et al. The pulmonary endothelial glycocalyx regulates neutrophil adhesion and lung injury during experimental sepsis. Nat Med. 2012;18:1217-23. 
12. Hansen TV, Vik A, Serhan CN. The protectin family of specialized proresolving mediators: potent immunoresolvents enabling innovative approaches to target obesity and diabetes. Front Pharmacol. 2018;9:1582.

13. Dalli J, Chiang N, Serhan CN. Identification of 14-series sulfido-conjugated mediators that promote resolution of infection and organ protection. Proc Natl Acad Sci U S A. 2014;111:E4753-4761.

14. Ramon S, Dalli J, Sanger JM, Winkler JW, Aursnes M, Tungen JE, Hansen TV, Serhan CN. The protectin PCTR1 Is produced by human M2 macrophages and enhances resolution of infectious inflammation. Am J Pathol. 2016;186:962-73.

15. Serhan CN, de la Rosa $X$, Jouvene $C$. Novel mediators and mechanisms in the resolution of infectious inflammation: evidence for vagus regulation. J Intern Med. 2019;286:240-58.

16. Dalli J, Colas RA, Arnardottir H, Serhan CN. Vagal regulation of group 3 innate lymphoid cells and the immunoresolvent PCTR1 controls infection resolution. Immunity. 2017:46:92-105.

17. Li H, Hao Y, Zhang H, Ying W, Li D, Ge Y, Ying B, Cheng B, Lian Q, Jin S. Posttreatment with Protectin DX ameliorates bleomycin-induced pulmonary fibrosis and lung dysfunction in mice. Sci Rep. 2017;7:46754.

18. Serhan CN. Pro-resolving lipid mediators are leads for resolution physiology. Nature. 2014;510:92-101.

19. Liu YJ, Li H, Tian Y, Han J, Wang XY, Li XY, Tian C, Zhang PH, Hao Y, Gao F, Jin SW. PCTR1 ameliorates lipopolysaccharide-induced acute inflammation and multiple organ damage via regulation of linoleic acid metabolism by promoting FADS1/FASDS2/ELOV2 expression and reducing PLA2 expression. Lab Invest. 2020;100:904-15.

20. Zhang PH, Han J, Cao F, Liu YJ, Tian C, Wu CH, Smith FG, Hao Y, Jin SW. PCTR1 improves pulmonary edema fluid clearance through activating the sodium channel and lymphatic drainage in lipopolysaccharideinduced ARDS. J Cell Physiol. 2020;235(12):9510-23.

21. Chelazzi C, Villa G, Mancinelli P, De Gaudio AR, Adembri C. Glycocalyx and sepsis-induced alterations in vascular permeability. Crit Care. 2015;19:26.

22. Schmidt EP, Overdier KH, Sun X, Lin L, Liu X, Yang Y, Ammons LA, Hiller TD, Suflita MA, Yu Y, et al. Urinary glycosaminoglycans predict outcomes in septic shock and acute respiratory distress syndrome. Am J Respir Crit Care Med. 2016;194:439-49.

23. Haeger SM, Yang Y, Schmidt EP. Heparan sulfate in the developing, healthy, and injured lung. Am J Respir Cell Mol Biol. 2016;55:5-11.

24. Joffre J, Hellman J, Ince C, Ait-Oufella H. Endothelial responses in sepsis. Am J Respir Crit Care Med. 2020;202:361-70.
25. Yang Y, Haeger SM, Suflita MA, Zhang F, Dailey KL, Colbert JF, Ford JA, Picon MA, Stearman RS, Lin L, et al. Fibroblast growth factor signaling mediates pulmonary endothelial glycocalyx reconstitution. Am J Respir Cell Mol Biol. 2017;56:727-37.

26. Gao R, Ma Z, Hu Y, Chen J, Shetty S, Fu J. Sirt1 restrains lung inflammasome activation in a murine model of sepsis. Am J Physiol Lung Cell Mol Physiol. 2015;308:L847-853.

27. Lipphardt M, Dihazi H, Muller GA, Goligorsky MS. Fibrogenic secretome of sirtuin 1-deficient endothelial cells: Wnt Notch and Glycocalyx Rheostat. Front Physiol. 2018;9:1325.

28. Fu C, Hao S, Xu X, Zhou J, Liu Z, Lu H, Wang L, Jin W, Li S. Activation of SIRT1 ameliorates LPS-induced lung injury in mice via decreasing endothelial tight junction permeability. Acta Pharmacol Sin. 2019:40:630-41.

29. Tilstra JS, Clauson CL, Niedernhofer LJ, Robbins PD. NF-kappaB in aging and disease. Aging Dis. 2011;2:449-65.

30. Kauppinen A, Suuronen T, Ojala J, Kaarniranta K, Salminen A. Antagonistic crosstalk between NF-kappaB and SIRT1 in the regulation of inflammation and metabolic disorders. Cell Signal. 2013;25:1939-48.

31. Yeung F, Hoberg JE, Ramsey CS, Keller MD, Jones DR, Frye RA, Mayo MW. Modulation of NF-kappaB-dependent transcription and cell survival by the SIRT1 deacetylase. EMBO J. 2004;23:2369-80.

32. An X, Zhang L, Yao Q, Li L, Wang B, Zhang J, He M, Zhang J. The receptor for advanced glycation endproducts mediates podocyte heparanase expression through NF-kappaB signaling pathway. Mol Cell Endocrinol. 2018;470:14-25.

33. Ramani VC, Vlodavsky I, Ng M, Zhang Y, Barbieri P, Noseda A, Sanderson $\mathrm{RD}$. Chemotherapy induces expression and release of heparanase leading to changes associated with an aggressive tumor phenotype. Matrix Biol. 2016;55:22-34.

34. Amado-Azevedo J, van Stalborch AD, Valent ET, Nawaz K, van Bezu J, Eringa EC, Hoevenaars FPM, De Cuyper IM, Hordijk PL, van Hinsbergh VWM, et al. Depletion of Arg/Abl2 improves endothelial cell adhesion and prevents vascular leak during inflammation. Angiogenesis. 2021. https://doi.org/10.1007/s10456-021-09781-x.

\section{Publisher's Note}

Springer Nature remains neutral with regard to jurisdictional claims in published maps and institutional affiliations.
Ready to submit your research? Choose BMC and benefit from:

- fast, convenient online submission

- thorough peer review by experienced researchers in your field

- rapid publication on acceptance

- support for research data, including large and complex data types

- gold Open Access which fosters wider collaboration and increased citations

- maximum visibility for your research: over $100 \mathrm{M}$ website views per year

At BMC, research is always in progress.

Learn more biomedcentral.com/submissions 